\title{
EXISTENCE AND UNIQUENESS OF SOLUTIONS OF ORDINARY DIFFERENTIAL EQUATIONS
}

\author{
DAVID V. V. WEND
}

1. Introduction. This paper is concerned with the existence and uniqueness of solutions of initial value problems for systems of ordinary differential equations under various monotonicity conditions. Let

$$
y^{\prime}=f(x, y), \quad y\left(x_{0}\right)=y_{0}
$$

Here $x$ is a real scalar, $y, y_{0}$ and $f$ are real $n$-vectors, and $f$ is defined in a region $B$ determined by the inequalities

$$
x_{0} \leqq x \leqq x_{0}+a, \quad\left\|y-y_{0}\right\| \leqq b \quad(a>0, b>0),
$$

where $\|y\|$ is any norm.

For two vectors $y=\left(y_{k}\right)$ and $z=\left(z_{k}\right)$ define $y \leqq z(y<z)$ if, and only if, $y_{k} \leqq z_{k}\left(y_{k}<z_{k}\right), k=1, \cdots, n$. We say $f(x, y)=\left(f_{k}(x, y)\right)$ is nondecreasing in $x$ if $f(x, y) \leqq f\left(x^{\prime}, y\right)$ whenever $(x, y)$ and $\left(x^{\prime}, y\right)$ are in $B$ and $x<x^{\prime}$, and $f(x, y)$ is nondecreasing in $y$ if $f(x, y) \leqq f(x, z)$ whenever $(x, y)$ and $(x, z)$ are in $B$ and $y \leqq z$, while $f(x, y)$ is quasi-nondecreasing in $y$ if for each $k=1, \cdots n, f_{k}(x, y) \leqq f_{k}(x, z)$ whenever $y \leqq z$ and $y_{k}=z_{k}$. Finally, we say $y(x)$ is a strictly increasing function of $x$ if $y(x)<y\left(x^{\prime}\right)$ whenever $x<x^{\prime}$.

In $\$ 2$ the existence to the right of $x=x_{0}$ of a solution in the extended sense of (1.1) is proved under the assumptions $f(x, y) \geqq 0$ and $f(x, y)$ is nondecreasing in both $x$ and $y$. Then it is shown that (1.1) has at most one solution to the right of $x=x_{0}$ under the assumptions that $f(x, y)$ is nondecreasing in $x$ and quasi-nondecreasing in $y$ and satisfies an additional condition which prevents any component of a solution from remaining constant. $\$ 3$ is devoted to examples illustrating the necessity of some of the hypotheses in the existence and uniqueness theorems.

The uniqueness theorem of $\$ 2$ generalizes results obtained earlier by the author [3], [4].

2. Existence and uniqueness theorems. Let $B$ be the $(n+1)$ dimensional region given by (1.2) and let $B^{\prime}$ be the subregion of $B$ where $y \geqq y_{0}$. By a solution of (1.1) will be meant a solution in the extended sense [1, p. 42].

Presented to the Society, January 23, 1968; received by the editors July 18, 1968. 
THEOREM 2.1. Suppose the function $f(x, y)$ in (1.1) is defined in the region $B$ given by (1.2). If in addition $f(x, y) \geqq 0$ in $B^{\prime}$ and $f(x, y)$ is nondecreasing in both $x$ and $y$ in $B^{\prime}$, then there exists a solution of the initial value problem (1.1) to the right of $x=x_{0}$.

Proof. We show that the method of successive approximations yields a solution to the right of $x=x_{0}$.

By a translation if necessary, we may assume without loss of generality that $x_{0}=0$. Let $M=\max \|f(x, y)\|$ in $B^{\prime}$ and $\delta=\min (a, b / M)$. Define $g_{0}(x)=y_{0}, g_{s}(x)=y_{0}+\int_{0}^{x} f\left(t, g_{s-1}(t)\right) d t, s=1,2,3, \ldots$. Clearly $g_{0}(x)$ is nondecreasing and $\left(x, g_{0}(x)\right)$ lies in $B^{\prime}$ for $0 \leqq x \leqq \delta$. Assume for $m=1,2, \cdots, s-1$ that for $0 \leqq x \leqq \delta, g_{m}(x)$ is nondecreasing, $g_{m}(x)$ $\geqq g_{m-1}(x)$ and $\left(x, g_{m}(x)\right)$ lies in $B^{\prime}$. Since $f(x, y)$ is nondecreasing in $x$ and $y$ in $B^{\prime}$, it follows that $f\left(x, g_{s-1}(x)\right)$ is nondecreasing, hence integrable, and $f\left(x, g_{s-1}(x)\right) \geqq 0$ on $[0, \delta]$. Therefore $g_{s}(x)$ exists and is nondecreasing, and $g_{s}(x) \geqq g_{s-1}(x)$ on $[0, \delta]$. Also, $\left\|g_{s}(x)-y_{0}\right\| \leqq \delta M \leqq b$, so $\left(x, g_{s}(x)\right)$ lies in $B^{\prime}$ for $0 \leqq x \leqq \delta$.

Since $g_{0}(x) \leqq g_{1}(x) \leqq \cdots$ and $\left\|g_{s}(x)\right\| \leqq\left\|y_{0}\right\|+b$ for each $s$, it follows that $G(x)=\lim _{s \rightarrow \infty} g_{s}(x)$ exists and is nondecreasing on $[0, \delta]$. Also, $f\left(x, g_{0}(x)\right) \leqq f\left(x, g_{1}(x)\right) \leqq \cdots \leqq f(x, G(x))$ and $\|f(x, G(x))\| \leqq M$, so it follows from the Lebesgue monotone convergence theorem that

$$
G(x)=y_{0}+\int_{0}^{x}\left(\lim _{s \rightarrow \infty} f\left(t, g_{s}(t)\right)\right) d t=y_{0}+\int_{0}^{x} f(t, G(t)-0) d t .
$$

By $f(t, G(t)-0)$ we mean the limit of $f(t, y)$ as $y$ increases to $G(t)$ in $B^{\prime}$, where it is understood that, with $G(t)=\left(G_{k}(t)\right)$, for each $k=1, \cdots, n$ if $G_{k}(t)$ is greater than the $k$ th component of $y_{0}$, then the $k$ th component of $y$ increases strictly to $G_{k}(t)$, while if $G_{k}(t)$ equals the $k$ th component of $y_{0}$, then the $k$ th component of $y$ is kept equal to $G_{k}(t)$.

It remains to show that $G^{\prime}(x)=f(x, G(x))$ a.e. on $[0, \delta]$. Since $f(t, G(t)-0)$ is nondecreasing, each $G_{k}(x)$ is absolutely continuous and therefore $G^{\prime}(x)=f(x, G(x)-0)$ a.e., so it suffices to show $f(x, G(x)-0)$ $=f(x, G(x))$ a.e. Suppose this is not the case. Let $H(x)=\left(H_{k}(x)\right)$ $=f(x, G(x)-0), F(x)=\left(F_{k}(x)\right)=f(x, G(x))$. Clearly $H(x) \leqq F(x)$, so, since $H(x)$ and $F(x)$ are continuous a.e., being monotone, there is an $x_{0}, 0<x_{0}<\delta$, such that $H(x)$ and $F(x)$ are continuous at $x=x_{0}$ and at least one component of $H(x)$ is strictly less than the corresponding component of $F(x)$ at $x_{0}$, say $H_{k}\left(x_{0}\right)<F_{k}\left(x_{0}\right)$. By continuity it follows that $H_{k}(x)<F_{k}\left(x_{0}\right)$ immediately to the right of $x_{0}$. For each $k, G_{k}(x)$ is strictly increasing unless $G_{k}(t)$ equals identically the $k$ th component 
of $y_{0}, 0<t \leqq x$, since $G_{k}^{\prime}(x)=f_{k}(x, G(x)-0)>0$ a.e. unless $f_{k}(t, G(t)-0)$ $\equiv 0,0<t \leqq x$, in which case $f_{k}\left(t, g_{s}(t)\right) \equiv 0, s=0,1,2, \cdots$. But then for $x>x_{0}, x$ sufficiently close to $x_{0}$, this leads to the contradiction $H_{k}(x)<F_{k}\left(x_{0}\right) \leqq H_{k}(x)$.

NoTE. If $f(x, y) \geqq 0$ and $f(x, y)$ is nondecreasing in both $x$ and $y$ in all of $B$, then the solution obtained above is the minimal solution to the right of $x=x_{0}$.

THEOREM 2.2. Assume the function $f$ in (1.1) is defined in $B$ and

(a) $f(x, y)$ is nondecreasing in $x$ and quasi-nondecreasing in $y$ in $B^{\prime}$ and

(b) for any solution $y(x)$ of (1.1), $f(x, y(x))>0$ for $x>x_{0}$. Then the initial value problem (1.1) has at most one solution to the right of $x=x_{0}$.

The author would like to thank A. A. Kayande and V. Lakshmikantham for suggesting the present form of this theorem.

Proof. Assume (1.1) has two solutions $y(x)=\left(y_{1}(x), \cdots, y_{n}(x)\right)$ and $z(x)=\left(z_{1}(x), \cdots, z_{n}(x)\right)$ defined on the common interval $\left[x_{0}, x_{0}+c\right], 0<c \leqq a$. It follows from condition (b) that $y(x)$ and $z(x)$ are strictly increasing functions of $x$ on $\left[x_{0}, x_{0}+c\right]$. Let $y_{0}$ $=\left(a_{1}, \cdots, a_{n}\right)$ and

$$
\begin{aligned}
N=\min \left(y_{1}\left(x_{0}+c\right)-a_{1}, \cdots, y_{n}\left(x_{0}+c\right)-a_{n},\right. \\
\\
\left.z_{1}\left(x_{0}+c\right)-a_{1}, \cdots, z_{n}\left(x_{0}+c\right)-a_{n}\right) .
\end{aligned}
$$

For each $k=1, \cdots, n$ there exist numbers $d_{k}$ and $e_{k}$ such that $y_{k}\left(x_{0}+d_{k}\right)=z_{k}\left(x_{0}+e_{k}\right)=N+a_{k}, 0<d_{k}, e_{k} \leqq c$. Let

$$
d=\min \left(d_{1}, \cdots, d_{n}, e_{1}, \cdots, e_{n}\right) .
$$

Then for each $k=1, \cdots, n, y_{k}(x) \leqq a_{k}+N$ and $z_{k}(x) \leqq a_{k}+N$ on $\left[x_{0}, x_{0}+d\right]$.

Suppose without loss of generality that for some $x_{1}, x_{0}<x_{1} \leqq x_{0}+d$, a component of $y\left(x_{1}\right)$ is less than the corresponding component of $z\left(x_{1}\right)$. On $\left[x_{0}, x_{0}+d\right]$ let functions $v_{k}(x)$ be defined by

$$
z_{k}\left(v_{k}(x)\right)=y_{k}(x), \quad k=1, \cdots, n .
$$

Since each component of $y(x)$ and $z(x)$ is strictly increasing and absolutely continuous, it follows that each $v_{k}(x)$ is strictly increasing and absolutely continuous, $v_{k}\left(x_{0}\right)=x_{0}$ and $v_{k}^{\prime}(x)$ exists almost everywhere on $\left[x_{0}, x_{0}+d\right]$. Thus on this interval

$$
v_{k}^{\prime}(x)=z_{k}^{\prime}\left(v_{k}(x)\right) v_{k}^{\prime}(x) \quad \text { a.e., } \quad k=1, \cdots n .
$$


Noting that $v_{k}\left(x_{1}\right)<x_{1}$ if $y_{k}\left(x_{1}\right)<z_{k}\left(x_{1}\right)$, it follows that there exists $x^{\prime}, x_{0}<x^{\prime}<x_{1}$, and an integer $j, 1 \leqq j \leqq n$, such that $v_{j}\left(x^{\prime}\right)<x^{\prime}, v_{j}\left(x^{\prime}\right)$ $\leqq v_{k}\left(x^{\prime}\right), k=1, \cdots, n$, and $v_{j}^{\prime}\left(x^{\prime}\right), y_{j}^{\prime}\left(x^{\prime}\right)$ and $z_{j}^{\prime}\left(v_{j}\left(x^{\prime}\right)\right)$ exist. Using (2.1) and conditions (a) and (b),

$$
\begin{aligned}
0<y_{j}^{\prime}\left(x^{\prime}\right) & =f_{j}\left(v_{j}\left(x^{\prime}\right), z_{1}\left(v_{j}\left(x^{\prime}\right)\right), \cdots, z_{n}\left(v_{j}\left(x^{\prime}\right)\right)\right) v_{j}^{\prime}\left(x^{\prime}\right) \\
& \leqq f_{j}\left(x^{\prime}, z_{1}\left(v_{1}\left(x^{\prime}\right)\right), \cdots, z_{j}\left(v_{j}\left(x^{\prime}\right)\right), \cdots, z_{n}\left(v_{n}\left(x^{\prime}\right)\right)\right) v_{j}^{\prime}\left(x^{\prime}\right) \\
& =y_{j}^{\prime}\left(x^{\prime}\right) v_{j}^{\prime}\left(x^{\prime}\right) .
\end{aligned}
$$

Hence $v_{j}^{\prime}\left(x^{\prime}\right) \geqq 1$.

For each $x, x_{0} \leqq x \leqq x_{0}+d$, let $v(x)=\min \left(v_{1}(x), \cdots, v_{n}(x)\right)$. It is easily verified that $v(x)$ is absolutely continuous; also, if $v^{\prime}(x)$ and $v_{k}^{\prime}(x)$ exist and $v(x)=v_{k}(x)$, then $v^{\prime}(x)=v_{k}^{\prime}(x)$. Let

$$
\begin{array}{r}
x^{*}=\operatorname{glb}\left(x: x_{0} \leqq x \leqq x^{\prime} \text { and for each } t, \quad x \leqq t \leqq x^{\prime},\right. \text { there } \\
\text { exists } \left.k, \quad 1 \leqq k \leqq n \text {, such that } y_{k}(t)<z_{k}(t)\right) .
\end{array}
$$

Then $x_{0} \leqq x^{*}<x^{\prime}$. By the above argument, $v^{\prime}(x) \geqq 1$ a.e. on $\left[x^{*}, x^{\prime}\right]$, hence, since $v(x)$ is absolutely continuous on this interval,

$$
r^{\prime}\left(x^{*}\right) \leqq v\left(x^{\prime}\right)+\left(x^{*}-x^{\prime}\right)=v_{j}\left(x^{\prime}\right)-x^{\prime}+x^{*}<x^{*} .
$$

But this implies that for some $k, v\left(x^{*}\right)=v_{k}\left(x^{*}\right)$ and $y_{k}\left(x^{*}\right)=z_{k}\left(v_{k}\left(x^{*}\right)\right)$ $<z_{k}\left(x^{*}\right)$, so $x^{*}>x_{0}$ and $y_{k}(x)<z_{k}(x)$ immediately to the left of $x^{*}$, contrary to the definition of $x^{*}$. This contradiction proves the theorem.

Corollary 2.3. Assume the function $f$ in (1.1) is defined in $B$ and that

(a) $f(x, y)$ is nondecreasing in both $x$ and $y$ in $B^{\prime}$ and $f(x, y) \geqq 0$ in $B$ and

(b) $f\left(x, y_{0}\right)>0$ for $x_{0}<x \leqq x_{0}+a$.

Then the initial value problem (1.1) has a unique solution to the right of $x=x_{0}$.

Proof. Theorem 2.1 guarantees the existence of a solution to the right of $x=x_{0}$. Condition (a) of this corollary clearly implies condition (a) of Theorem 2.2.

Let $y(x)$ be a solution of (1.1). Then

$$
y(x)=y_{0}+\int_{x_{\dot{v}}}^{x} f(t, y(t)) d t \geqq y_{0} .
$$

Hence $f(x, y(x)) \geqq f\left(x, y_{0}\right)>0$ for $x>x_{0}$, so condition (b) of Theorem 2.2 is satisfied. 
Corollary 2.4 (SeE [3]). Let

$$
\begin{array}{r}
u^{(n)}=F\left(x, u, u^{\prime}, \cdots, u^{(n-1)}\right), \quad u^{(k)}\left(x_{0}\right)=c_{k}, \\
k=0,1, \cdots, n-1, \quad(u \text { and } F \text { scalars }) .
\end{array}
$$

Let $R$ be the region defined by the inequalities $x_{0} \leqq x \leqq x_{0}+a,\left|s_{j}-c_{j}\right| \leqq b$, $j=0,1, \cdots, n-1(a>0, b>0)$. Suppose $F\left(x, s_{0}, \cdots, s_{n-1}\right)$ is defined in $R$ and in addition

(a) $F$ is nonnegative and nondecreasing in each of $x, s_{0}, \cdots, s_{n-1}$ in $R$;

(b) $F\left(x, c_{0}, \cdots, c_{n-1}\right)>0$ for $x_{0}<x \leqq x_{0}+a$;

(c) $c_{k} \geqq 0, k=1,2, \cdots, n-1$.

Then the initial value problem (2.2) has a unique solution in $R$.

Proof. The Wronskian system equivalent to $(2.2)$ is

$$
y^{\prime}=f(x, y), \quad y\left(x_{0}\right)=y_{0} .
$$

where

$y_{k}=u^{(k-1)}, k=1, \cdots, n, f(x, y)=\left(y_{2}, y_{3}, \cdots, y_{n}, F\left(x, y_{1}, \cdots, y_{n}\right)\right)$, and

$$
y_{0}=\left(c_{0}, c_{1}, \cdots, c_{n-1}\right) .
$$

It follows from conditions (a) and (c) above and Theorem 2.1 that (2.3) has a solution $y(x)$ to the right of $x=x_{0}$. Condition (a) in Theorem 2.2 is clearly satisfied for (2.3). Conditions (a) and (c) show that in succession each $y_{n-k}(x)$ is nonnegative and nondecreasing, $k=0,1, \cdots, n-2$, while $y_{1}(x) \geqq c_{0}$ and $y_{1}(x)$ is nondecreasing. But then conditions (a), (b) and (c) imply that in succession

$$
y_{n-k}(x)>0 \quad \text { for } x>x_{0}, \quad k=0,1, \cdots, n-2,
$$

and

$$
F\left(x, y_{1}(x), \cdots, y_{n}(x)\right) \geqq F\left(x, c_{0}, \cdots, c_{n-1}\right)>0 \quad \text { for } x>x_{0} .
$$

Thus condition (b) of Theorem 2.2 is satisfied. Hence (2.3) has a unique solution in $R$, and the same is therefore true for (2.2).

Note. If in the scalar case $(n=1)$ of Theorem $2.2, f\left(x_{0}, y_{0}\right)>0$, then, since each component of a solution is absolutely continuous, the argument used in $[2$, p. 34 , proof of Theorem 6.2] can be applied to $d x / d y=1 / f(x, y), x\left(y_{0}\right)=x_{0}$, to prove the theorem in this case.

3. Some examples. In this section we give examples showing the 
necessity of some of the hypotheses in the theorems and corollaries in $\$ 2$.

The first examples refer to Theorem 2.1. Let

$$
y^{\prime}=f(x, y), \quad y(0)=0 \quad(y, f \text { scalars }),
$$

and let $B$ be the region determined by the inequalities

$$
0 \leqq x \leqq 1, \quad-1 \leqq y \leqq 1 .
$$

Suppose first that in $B, f(x, y)=2$ for $y \leqq x, f(x, y)=1$ for $x<y$. Here $f(x, y)$ is nonnegative and nondecreasing in $x$ but not nondecreasing in $y$, and (3.1) has no solution in $B$ for this $f(x, y)$. It is interesting to note that the successive approximations are alternately $x$ and $2 x$.

In the next example the function $f(x, y)$ in (3.1) is nonnegative and nondecreasing in $y$ but not nondecreasing in $x$, and for this $f(x, y)$ the problem (3.1) has no solution. Let $S$ be a subset of $[0,1]$ such that $S \cap[0, x]$ is nonmeasurable for each $x, 0<x \leqq 1$. In $B$ let $f(x, y)$ $=0$ if $x \in S$ and $f(x, y)=1$ otherwise.

The next examples are concerned with the uniqueness of solutions of (3.1) in the region $B$ determined by (3.2).

Let $f(x, y)=1$ for $y \leqq x, f(x, y)=2$ for $x<y$ in $B$. The minimal solution is $y=x$, the maximal solution is $y=2 x$. Here $f(x, y)$ is not nondecreasing in $x$; otherwise all hypotheses in Theorem 2.2 are satisfied.

For the next example let $f(x, y)=0$ for $y \leqq 0, f(x, y)=1$ for $0<y$ in $B$. Again there are many solutions: e.g. $y(x)=0$ and $y(x)=x$. Here $f$ is nonnegative and nondecreasing in both $x$ and $y$ but $f(x, 0)=0$, so condition (b) of Theorem 2.2 is not fulfilled by the zero solution. In. Corollary 2.4 conditions (a) and (c) are fulfilled but not condition (b).

Next let $f(x, y)=0$ if $y=1 / n, n=1,2,3, \cdots, f(x, y)=1$ otherwise in $B$. The maximal solution is $y(x)=x$. Another solution is given by

$$
\begin{array}{rlrl}
y(x) & =x-1 / n(n+1), & & 1 / n \leqq x \leqq 1 / n(n+1)+1 / n, \\
& n=2,3, \cdots, \\
& =1 / n, & & n=n(n+1)+1 / n \leqq x \leqq 1 /(n-1), \\
& =0 & & x=0 .
\end{array}
$$

In this example there is no minimal solution. Here $f(x, 0)=1$ for $x \geqq 0$ and $f(x, y)$ is nondecreasing in $x$ but not nondecreasing in $y$. This shows that in Corollary 2.3 the condition " $f(x, y)$ is nondecreasing in $y$ " cannot be relaxed to the condition " $f(x, y)$ is quasi-nondecreasing in $y . "$ 
Finally suppose $f(x, y)=-1$ for $y<0$ and $f(x, y)=1$ for $0 \leqq y$. Then $y(x)=x$ and $y(x)=-x$ are solutions. Again condition (b) of Theorem 2.2 is not satisfied, while in Corollary 2.4 all hypotheses are fulfilled except the condition $f(x, y)$ be nonnegative in $R$. See [3] for other examples.

\section{REFERENCES}

1. E. A. Coddington and N. Levinson, Theory of ordinary differential equations, McGraw-Hill, New York, 1955.

2. P. Hartman, Ordinary differential equations, Wiley, New York, 1964.

3. D. V. V. Wend, Uniqueness of solutions of ordinary differential equations, Amer. Math. Monthly 74 (1967), 948-950.

4. - - Existence and uniqueness of solutions of ordinary differential equations, Notices Amer. Math. Soc. 15 (1968), 89.

Montana State University 